\title{
Childhood arterial ischemic stroke in Egypt
}

\begin{abstract}
Objective: Arterial ischemic stroke (AIS) in childhood is a serious disorder about which little is published in Egypt. The aim of this study is to determine the epidemiology and outcome of AIS in Egyptian children.

Methods: Cases of childhood AIS, admitted to the EL Mataryia Teaching Hospital, Cairo, Egypt 2004-2008, were identified by medical record search. Information was collected on demographics, risk factors, arterial distribution, results of thrombophilic testing, management and outcome.

Results: During the 5 years of review 87 patients presented with 106 episodes of AIS. Children less than 12 months of age constitute more than one third of all cases. Identifiable risk factors were present in $66 \%$ of cases, congenital heart diseases was the major risk factor. Thrombophilic testing was incomplete with initial abnormalities present in $17 \%$ of cases tested. The estimated stroke-related mortality was $6.8 \%$. Of the patients who survived and who had follow-up details available, $73 \%$ had a neurological deficit. 48 patients $(55 \%)$ received anticoagulation. There was no statistically significant association between treatment with anticoagulation and normal neurological outcome.

Conclusion: AIS is over-represented in children less than 12 months of age and results in death or residual neurological impairment in the majority of cases. Further prospective studies are needed to identify risk factors for poor outcome. New prospective studies are needed to provide important information on clinical and laboratory based risk factors and to improve the outcome of childhood AIS.
\end{abstract}

\author{
Nabil Kitchener,' Magdy Khalaf,' Gamal \\ Khairalla,' Mohammed Helmy' \\ 'Department of Neuropsychiatry, El-Mataryia Teaching Hospital, \\ General Organization for Teaching Hospitals and Institutes, \\ Egypt \\ ${ }^{2}$ Department of Pediatrics, El-Mataryia Teaching Hospital, \\ General Organization for Teaching Hospitals and Institutes, \\ Egypt
}

Correspondence: Nabil Kitchener, Department of Neuropsychiatry, El-Mataryia Teaching Hospital, General Organization for Teaching Hospitals and Institutes, Egypt, Emial nabilkitchener@consultant.com

Received: September 09, 2017| Published: March 23, 2018

\section{Introduction}

Arterial ischemic stroke (AIS) in neonates and children is a major cause of significant morbidity and mortality. ${ }^{1-4}$ Although considered rare, estimates place the incidence of AIS at 2.7 per 100 000 children, which is comparable to the incidence of brain tumors in children. ${ }^{5}$ Unlike brain tumors however, there is no comprehensive, multi-institutional approach to understand the pathophysiology and improve the management of childhood AIS. Our understanding of the epidemiology of childhood AIS in Egypt is limited. Judging by the largest cohort of childhood stroke available, the majority of children with AIS presented in association with a recognized risk factor. ${ }^{6}$ However, over one third of cases occur in otherwise well children. ${ }^{6}$ Recent interests have focused on thrombophilia as a cause of AIS in these children. ${ }^{7}$ Thrombophilic states may lead to a reduced threshold for pathological thrombus formation in cerebral vessels presenting with AIS in children. ${ }^{8}$ An association between thrombophilia and AIS may warrant development of primary and secondary thromboprophylactic strategies as prevention of childhood AIS. To gain a greater understanding of epidemiology, risk factors including thrombophilic abnormalities, and outcome of childhood AIS in Egypt, we reviewed all cases of AIS admitted to the EL Mataryia Teaching Hospital, Cairo, Egypt 2004-2008.

\section{Methods}

Cases were identified by a medical record search. Medical records were reviewed identifying eligible patients. Eligibility required a radiological diagnosis of arterial ischemic stroke, radiological tests include: Computed Tomography (CT) scans, Magnetic Resonance Imaging (MRI), Magnetic Resonance Angiogram (MRA) or Cerebral Angiogram and echocardiogram. Cases that did not have radiological demonstration of arterial ischemic stroke were excluded. Medical records of eligible patients were analyzed and data collected on the following: demographics, physical examination including a neurological examination, risk factors, arterial distribution, results of thrombophilic testing, management and outcome.

\section{Results}

\section{Patient population and demographics}

One hundred and thirty-six patients were identified using the ICD codes. After medical record review, (87 patients with 106 episodes) met the eligibility criteria of radiographic proven AIS (39 females and 48 males). Twelve patients had one or more recurrent episodes of AIS. The mean age of presentation was 3.4 years (range 1 day to 15.1 years). Forty episodes (37.7\%) of AIS occurred in children less than 12 months of age with $12.2 \%$ of total episodes occurring in the perinatal period (Figure 1) (Table 1).

\section{Risk factors}

Risk factors for AIS were identified in 57 patients $(66 \%)$. Almost one third of all strokes were associated with congenital heart disease (Table 2).

\section{Arterial distribution}

The anterior cerebral circulation was involved in 89 episodes (57 episodes involving a single vessel distribution, 32 episodes involving multiple vessel distributions). The posterior circulation was the site of involvement in 17 episodes.

\section{Thrombophilic testing}

Thrombophilic testing was performed in 59 patients $(55.6 \%)$ at a 
mean duration of 29 days (range 1-96 days) after presentation. Results of thrombophilic markers were abnormal in ten of these patients $(17 \%$ of those patients tested). Two patients had combined protein $\mathrm{S}$ and protein $\mathrm{C}$ deficiency. Three patients had isolated protein $\mathrm{C}$ deficiency and one patient had isolated factor $\mathrm{V}$ Leiden mutation. Four patients were positive for the anticardiolipin antibodies. No retesting was performed. Family testing was performed on patients with positive results, and only father of one child showed combined protein $\mathrm{S}$ and protein $\mathrm{C}$ deficiency.

Table I Frequency of Acute Ischemic Stroke in different age groups

\begin{tabular}{ll}
\hline Age & Frequency \\
\hline 0-1 Years & 40 \\
1-2 Years & 3 \\
2-3 Years & 9 \\
3-4 Years & 2 \\
4-5 Years & 2 \\
5-6 Years & 11 \\
6-7Years & 2 \\
7-8Years & 1 \\
8-9 Years & 2 \\
9-10 Years & 3 \\
10-11 Years & 4 \\
11-12 Years & 2 \\
12-13 Years & 1 \\
13-14 Years & 2 \\
14-15 Years & 2 \\
15-16 Years & 1 \\
\hline
\end{tabular}

Table 2 Risk factors for arterial ischaemic stroke (AIS)

\begin{tabular}{ll}
\hline Risk factor & $\begin{array}{l}\text { Number of patients and } \\
\text { percentage }\end{array}$ \\
\hline Congenital heart disease & $27(31 \%)$ \\
No known risk factor & $30(34.5 \%)$ \\
CNS vascular malformation & $7(8 \%)$ \\
CNS or ENT infection & $6(6.9 \%)$ \\
Head trauma & $5(5.7 \%)$ \\
Recent intracranial surgery & $4(4.6 \%)$ \\
Indwelling central venous line & $4(4.6 \%)$ \\
Intracranial tumor & $3(3.5 \%)$ \\
sickle cell disease & $\mathrm{I}(1.2 \%)$ \\
\hline
\end{tabular}

Table 3 Neurological morbidity of childhood arterial ischemic stroke (AIS)

\begin{tabular}{ll}
\hline Type of Neurological deficit & Patients and percentage \\
\hline Hemiplegia/hemiparesis & $24(47 \%)$ \\
Seizures & $12(23.5 \%)$ \\
Developmental delay & $8(15.68 \%)$ \\
Ataxia & $7(13.72 \%)$ \\
\end{tabular}

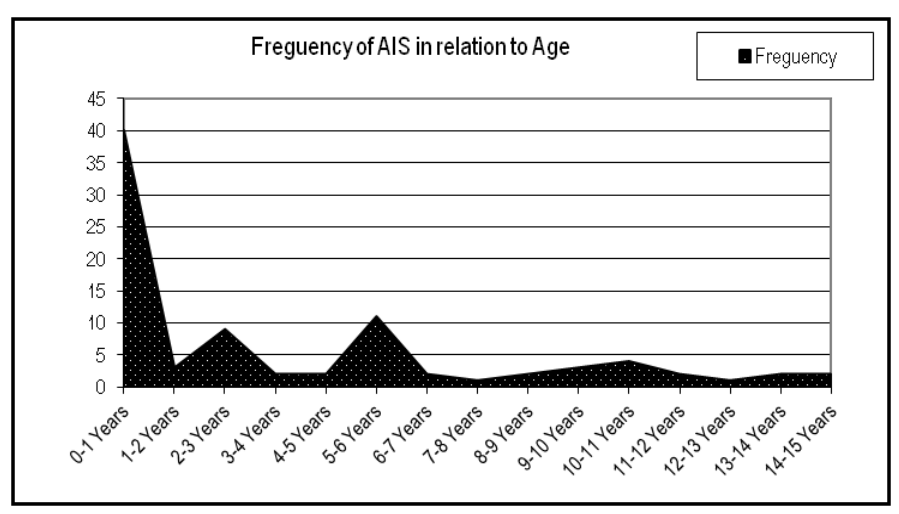

Figure I Frequency of Acute Ischemic Stroke in different age groups.

\section{Anticoagulation and outcome}

Patients were admitted to the Mataryia Teaching Hospital, for an average duration of 23.4 days (range 1-74 days). Forty eight patients had management that included anticoagulation (55\%). Twenty nine patients were treated with antiplatelet medication, ten patients were treated with both antiplatelet and anticoagulant medication and none received thrombolytic therapy. Seventeen patients died (19.5\%). Cause of death was attributed to AIS in six cases (estimated stroke related mortality of $6.8 \%$ ). Other causes of death included overwhelming sepsis, and cardio-respiratory arrest. Of the remainder, follow-up details were available for 60 patients $(85.7 \%)$ with a median followup period of 2.1 years (range 16 days to 6.8 years). Nineteen patients $(27 \%)$ were considered normal. Fifty-one patients $(73 \%)$ had an identifiable neurological deficit as shown in Table 3. Of the patients assessed as neurologically normal at follow up, twelve were managed with anticoagulation (four antiplatelet, and three antiplatelet and anticoagulant). This compared to 36 patients who had neurological deficits who were treated with anticoagulation. A chi square test showed no statistically significant relationship between treatment with anticoagulation and normal neurological outcome.

\section{Discussion}

Our study confirms the significant morbidity and mortality associated with childhood AIS in Egypt. A large proportion of childhood AIS occurs in children less than 12 months of age. Most children survive but with significant neurological deficits. Our results provide basic epidemiological data and support the establishment of prospective, multi-institutional stroke registries aimed at identifying important regional differences and prognostic factors in children presenting with AIS. Stroke registries are in turn necessary to provide information and assess feasibility for future multinational intervention trials aimed at improving the outcome and prevention of childhood AIS. Identifiable risk factors were present in about two thirds of our cases. Almost one third of cases presented with AIS in association with congenital cardiac disease, which is a well-recognized risk factor. ${ }^{8}$ One of the cases had sickle cell disease, a common association with AIS. No clinically significant conclusion can be drawn from the results of thrombophilic testing in our case series. Testing was incomplete with only one third of patients tested. The testing was performed at different times following the episode of AIS.

Management of AIS in our case series included anticoagulation for a significant proportion of cases. There are no evidence based guidelines on the treatment of AIS in children, ${ }^{9}$ and the use of anticoagulation 
or lytic therapy is controversial. ${ }^{10}$ The use of antithrombotic therapy in adult patients with AIS has also been extensively investigated. The benefits of antithrombotic therapy in AIS are matched with an increase in intracranial haemorrhage in the older population such that anticoagulation with heparin is no longer recommended for the acute management of AIS. ${ }^{11,12}$ However, the adult cerebral circulation may be at greater risk of hemorrhage compared to that of children and antithrombotic medication may be useful to improve the outcome of children with AIS. ${ }^{13}$ Finally, lytic therapy offers a potentially effective form of therapy for acute AIS with early reperfusion of cerebral tissue. ${ }^{13,14}$ Experience from adult studies shows lytic therapy needs to be given within the first 6 hours of symptoms and children often present significantly later. The present case series demonstrates that childhood AIS is associated with an estimated disease related mortality of $6.8 \%$ and almost $73 \%$ of survivors have significant neurological deficits. ${ }^{15,16}$ However, no clinical trials have been attempted to improve stroke outcome in children. Our results also confirm that thrombophilic testing in this population is problematic and the clinical significance of the screening is unknown. The Australian and New Zealand Stroke and Thrombophilia Registry has been established and incorporates most major tertiary pediatric hospitals in Australia and New Zealand. The registry is prospectively collecting clinical and laboratory information on childhood AIS and cerebral sinus venous thrombosis (SVT) and will perform centralized thrombophilic testing on all children enrolled. ${ }^{17}$ The registry will provide baseline information on risk factors and prognostic information on Australian and New Zealand children and an international clinical trial aimed at improving the outcome of childhood stroke is now running, Egypt is one site for this clinical trial.

\section{Conclusion}

AIS is over-represented in children less than 12 months of age and results in death or residual neurological impairment in the majority of cases. Further prospective studies are needed to identify risk factors for poor outcome. New prospective studies are needed to provide important information on clinical and laboratory based risk factors and to improve the outcome of childhood AIS.

\section{Acknowledgements}

None.

\section{Conflict of interest}

The author declares no conflict of interest.

\section{References}

1. Broderick J, Talbot GT, Prenger E, et al. Stroke in children within a major metropolitan area: the surprising importance of intracerebral hemorrhage. J Child Neurol. 1993;8(3):250-5.
2. Chabrier S, Husson B, Lasjaunias P, et al. Stroke in childhood: outcome and recurrence risk by mechanism in 59 patients. $J$ Child Neurol. 2000;15:290-4.

3. DeVeber GA, MacGregor D, Curtis R, et al. Neurologic outcome in survivors of childhood arterial ischemic stroke and sinovenous thrombosis. J Child Neurol. 2000;15(5):316-24.

4. Ganesan V, Hogan A, Shack N, et al. Outcome after ischaemic stroke in childhood. Dev Med Child Neurol. 2000;42(7):455-61.

5. Smith M, Gloeckler Reis L. Childhood Cancer: Incidence, Survival and Mortality. In: Pizzo P, Poplack D, editors. Principles and Practice of Pediatric Oncology. 14th ed. Philadelphia: Lippincott Williams \& Wilkins, 2002:1-121.

6. DeVeber G, Adams M, Andrew M. Canadian pediatric stroke registry Analysis III. Thrombo. Haemost. 1995:73.

7. Ganesan V, McShane MA, Liesner R, et al. Inherited prothrombotic states and ischaemic stroke in childhood. J Neurol Neurosurg Psychiatry. 1998;65(4):508-511.

8. Ganesan V, Prengler M, McShane M A, et al. Investigation of Risk Factors in Children with Arterial Ischemic Stroke. Ann Neurol. 2003;53(2):167-173.

9. Stewart-Wynne E. Stroke in the era of evidence based medicine: an Australian perspective. Aust Prescribe. 1999:22.

10. Atkinson RP, DeLemos C. Acute ischemic stroke management. Thrombosis Res. 2000;98(3):97-111.

11. Hacke W, Kaste M, Fieschi C, et al. Randomised double blind placebo controlled trial of thrombolytic therapy with intravenous alteplase in acute ischaemic stroke (ECASS II). Second European - Australasian Acute Stroke Study Investigators. Lancet. 1998;329(9136):1245-51.

12. Hankey G. Heparin in acute ischaemic stroke. The $\mathrm{T}$ wave is negative and it's time to stop. Med J Aust. 1998;169(10):534-6.

13. Michelson A, Bovill E, Monagle P, et al. Antithrombotic therapy in children. Chest. 1998;114(5 Suppl):S748-S69.

14. Hankey GJ, Warlow CP. Treatment and secondary prevention of stroke: evidence, costs, and effects on individuals and populations [see comments]. Lancet. 1999;354(9188):1457-63.

15. Bernarda TJ, Manco-Johnsonb MJ, Goldenbergb NA. The roles of anatomic factors, thrombophilia, and antithrombotic therapies in childhood-onset arterial ischemic stroke. Thromb Res. 2010;127(1):6-12.

16. Simma B, Lütschg J. Epidemiology and etiology of pediatric stroke. $J$ Ped Neurol. 2010;8(3):245-249.

17. Incecik F, Hergünera M O, Altunbasaka S. Risk factors and treatment outcomes for children with arterial ischemic stroke. J Clin Neuroscien. 2010;17(8):1000-1002. 